Collaborative EDNAP exercise on the IrisPlex system for DNA-based prediction of human eye colour

Chaitanya, L; Andersen, Jeppe Dyrberg; Morling, Niels; Kayser, Manfred

Published in:

Forensic Science International: Genetics

DOI:

10.1016/j.fsigen.2014.04.006

Publication date:

2014

Citation for published version (APA):

Chaitanya, L., Andersen, J. D., Morling, N., \& Kayser, M. (Ed.) (2014). Collaborative EDNAP exercise on the IrisPlex system for DNA-based prediction of human eye colour. Forensic Science International: Genetics, 11 , 241-251. https://doi.org/10.1016/j.fsigen.2014.04.006 


\section{Collaborative EDNAP exercise on the IrisPlex system for DNA-based prediction of human eye colour}

Lakshmi Chaitanya ${ }^{a, 1}$, Susan Walsh ${ }^{\mathrm{a}, 1,2}$, Jeppe Dyrberg Andersen ${ }^{\mathrm{b}}$, Ricky Ansell ${ }^{\mathrm{c}}$, Kaye Ballantyne $^{\mathrm{d}}$, David Ballard ${ }^{\mathrm{e}}$, Regine Banemann ${ }^{\mathrm{f}}$, Christiane Maria Bauer $^{\mathrm{g}}$, Ana Margarida Bento ${ }^{\mathrm{h}}$, Francesca Brisighelli ${ }^{\mathrm{i}}$, Tomas Capal ${ }^{\mathrm{j}}$, Lindy Clarisse ${ }^{\mathrm{k}}$, Theresa E. Gross ${ }^{1}$, Cordula Haas ${ }^{\mathrm{m}}$, Per Hoff-Olsen ${ }^{\mathrm{n}}$, Clémence Hollard ${ }^{\mathrm{o}}$, Christine Keyser $^{\circ}$, Kevin M. Kiesler ${ }^{\mathrm{p}}$, Priscila Kohler ${ }^{\mathrm{n}}$, Tomasz Kupiec ${ }^{\mathrm{q}}$, Adrian Linacre $^{\mathrm{r}}$, Anglika Minawi ${ }^{\mathrm{f}}$, Niels Morling ${ }^{\mathrm{b}}$, Helena Nilsson ${ }^{\mathrm{s}}$, Lina Norén ${ }^{c}$, Renée Ottens ${ }^{r}$, Jukka U. Palo ${ }^{t}$, Walther Parson ${ }^{g}$, Vincenzo L. Pascali ${ }^{\mathrm{i}}$, Chris Phillips ${ }^{\mathrm{u}}$, Maria João Porto ${ }^{\mathrm{h}}$, Antti Sajantila ${ }^{\mathrm{t}, \mathrm{v}}$, Peter M. Schneider ${ }^{1}$, Titia Sijen ${ }^{\mathrm{k}}$, Jens Söchtig ${ }^{\mathrm{u}}$, Denise Syndercombe-Court ${ }^{\mathrm{e}}$, Andreas Tillmar ${ }^{\mathrm{s}}$, Martina Turanska ${ }^{\mathrm{w}}$, Peter M. Vallone $^{\mathrm{p}}$, Lívia Zatkalíková ${ }^{\mathrm{w}}$, Anastassiya Zidkova ${ }^{\mathrm{x}, \mathrm{y}}$, Wojciech Branicki ${ }^{\mathrm{q}, \mathrm{z}}$, Manfred Kayser ${ }^{\mathrm{a}, *}$

\footnotetext{
a Department of Forensic Molecular Biology, Erasmus MC University Medical Centre Rotterdam, Rotterdam, The Netherlands

${ }^{\mathrm{b}}$ Section of Forensic Genetics, Department of Forensic Medicine, Faculty of Health Sciences, University of Copenhagen, Copenhagen, Denmark

' Swedish National Laboratory of Forensic Science (SKL), Linköping, Sweden

${ }^{\mathrm{d}}$ Forensic Services Department, Victoria Police, Macleod, Victoria, Australia

e Department of Forensic and Analytical Science, School of Biomedical Sciences, King's College London, United Kingdom

${ }^{\mathrm{f}}$ Kriminaltechnik, Bundeskriminalamt, Wiesbaden, Germany

${ }^{\mathrm{g}}$ Institute of Legal Medicine, Innsbruck Medical University, Innsbruck, Austria

${ }^{\mathrm{h}}$ Serviço de Genética e Biologia Forense, Delegação do Centro, Instituto Nacional de Medicina Legal, Coimbra, Portugal

i Forensic Genetics Laboratory, Institute of Legal Medicine, Università Cattolica del Sacro Cuore, Rome, Italy

${ }^{\mathrm{j}}$ Department of Forensic Genetics, Institute of Criminalistics, Prague, Czech Republic

${ }^{\mathrm{k}}$ Department of Human Biological Traces, Netherlands Forensic Institute, The Hague, The Netherlands

${ }^{1}$ Institute of Legal Medicine, Faculty of Medicine, University of Cologne, Cologne, Germany

${ }^{\mathrm{m}}$ Institute of Legal Medicine, University of Zurich, Zurich, Switzerland

${ }^{n}$ Department of Forensic Biology, Norwegian Institute of Public Health, Oslo, Norway

${ }^{\circ}$ Laboratoire d'Anthropologie Moléculaire, Université de Strasbourg, Institut de Médecine Légale, Strasbourg, France

p Material Measurement Laboratory, National Institute of Standards and Technology, Gaithersburg, MD, USA

${ }^{\mathrm{q}}$ Section of Forensic Genetics, Institute of Forensic Research, Kraków, Poland

${ }^{\mathrm{r}}$ School of Biological Sciences, Flinders University, Adelaide, South Australia, Australia

s Department of Forensic Genetics and Forensic Toxicology, National Board of Forensic Medicine, Linköping, Sweden

${ }^{\mathrm{t}}$ Department of Forensic Medicine, Hjelt Institute, University of Helsinki, Helsinki, Finland

${ }^{\mathrm{u}}$ Forensic Genetics Unit, Institute of Legal Medicine, University of Santiago de Compostela, Santiago de Compostela, Spain

${ }^{v}$ Institute of Applied Genetics, Department of Forensic and Investigative Genetics, University of North Texas Health Science Center, Fort Worth, TX, USA

${ }^{\mathrm{w}}$ Institute of Forensic Science, Ministry of the Interior, Department of Biology and DNA Analysis, Slovenská Lupca, Slovakia

${ }^{\mathrm{x}}$ Institute of Biology and Medical Genetics, First Faculty of Medicine, Charles University in Prague and General University Hospital in Prague, Prague, Czech Republic

${ }^{\mathrm{y}}$ Institute of Forensic Medicine and Toxicology, First Faculty of Medicine, Charles University in Prague, Prague, Czech Republic

${ }^{\mathrm{z}}$ Department of Genetics and Evolution, Institute of Zoology, Jagiellonian University, Kraków, Poland
}

\footnotetext{
* Corresponding author. Tel.: +31 107038073 ; fax: +31 107044575.

E-mail address: m.kayser@erasmusmc.nl (M. Kayser).

1 These authors contributed equally to this work.

${ }^{2}$ Current affiliation: Yale Molecular Anthropology Laboratory, Department of Anthropology, Yale University, 10 Sachem Street, New Haven, CT 06511, USA.
} 


\section{A R T I C L E I N F O}

\section{Article history:}

Received 27 December 2013

Received in revised form 24 March 2014

Accepted 11 April 2014

\section{Keywords:}

EDNAP

ISFG

IrisPlex

Eye colour prediction

Forensic DNA phenotyping

FDP

\begin{abstract}
A B S T R A C T
The IrisPlex system is a DNA-based test system for the prediction of human eye colour from biological samples and consists of a single forensically validated multiplex genotyping assay together with a statistical prediction model that is based on genotypes and phenotypes from thousands of individuals. IrisPlex predicts blue and brown human eye colour with, on average, $>94 \%$ precision accuracy using six of the currently most eye colour informative single nucleotide polymorphisms (HERC2 rs12913832, OCA2 rs1800407, SLC24A4 rs12896399, SLC45A2 (MATP) rs16891982, TYR rs1393350, and IRF4 rs12203592) according to a previous study, while the accuracy in predicting non-blue and non-brown eye colours is considerably lower. In an effort to vigorously assess the IrisPlex system at the international level, testing was performed by 21 laboratories in the context of a collaborative exercise divided into three tasks and organised by the European DNA Profiling (EDNAP) Group of the International Society of Forensic Genetics (ISFG). Task 1 involved the assessment of 10 blood and saliva samples provided on FTA cards by the organising laboratory together with eye colour phenotypes; $99.4 \%$ of the genotypes were correctly reported and $99 \%$ of the eye colour phenotypes were correctly predicted. Task 2 involved the assessment of 5 DNA samples extracted by the host laboratory from simulated casework samples, artificially degraded, and provided to the participants in varying DNA concentrations. For this task, $98.7 \%$ of the genotypes were correctly determined and $96.2 \%$ of eye colour phenotypes were correctly inferred. For Tasks 1 and 2 together, $99.2 \%$ (1875) of the 1890 genotypes were correctly generated and of the $15(0.8 \%)$ incorrect genotype calls, only $2(0.1 \%)$ resulted in incorrect eye colour phenotypes. The voluntary Task 3 involved participants choosing their own test subjects for IrisPlex genotyping and eye colour phenotype inference, while eye photographs were provided to the organising laboratory and judged; $96 \%$ of the eye colour phenotypes were inferred correctly across 100 samples and 19 laboratories. The high success rates in genotyping and eye colour phenotyping clearly demonstrate the reproducibility and the robustness of the IrisPlex assay as well as the accuracy of the IrisPlex model to predict blue and brown eye colour from DNA. Additionally, this study demonstrates the ease with which the IrisPlex system is implementable and applicable across forensic laboratories around the world with varying pre-existing experiences.
\end{abstract}

(c) 2014 Elsevier Ireland Ltd. All rights reserved.

\section{Introduction}

The field of forensic genetics is making great strides with the rapid scientific and technological evolution in obtaining new knowledge and creating innovative tools for solving crimes more and more effectively. Forensic DNA Phenotyping (FDP), a nascent advancement in this field, is one example of recent innovative developments in forensic genetics and involves the prediction of an individual's externally visible characteristics (EVCs) using biological samples obtained at a crime scene or from an anonymous body (parts) that may belong to a missing person [1-4]. Conventional DNA identification involves the comparison of DNA profiles derived from short tandem repeat (STR) marker genotypes obtained from evidence and reference samples, which is useful in cases when the sample donor is known from their DNA profile. In certain circumstances, sample donors may not be identified, i.e. a match (or familial match) of the DNA profiles with known suspects such as those in criminal offender DNA (profile) databases or with ante-mortem samples in cases of missing persons is not successful, or when DNA profile comparisons with putative relatives of missing persons does not reveal the degree of similarities indicating biological relationship. In these situations, FDP can be used to help investigative authorities focus their search for unknown suspects or missing persons towards individuals with particular DNA-predicted externally visible phenotypes. The DNAbased prediction of EVCs can thus aid investigations by police and other authorities by reducing the number of possible suspects or other individuals if conventional STR typing of the evidence fails to produce identification [1,2]. Furthermore, reconstructing appearance information from biological samples such as bones or teeth or other remains of deceased individuals is relevant in anthropological research disciplines including those relying on ancient DNA analysis $[1,2,5]$.
Several model-based approaches, amongst others [4,6], have been developed for predicting a particular phenotype from DNA most notably human eye (iris) colour [7]; the IrisPlex system is one such tool [8]. IrisPlex can accurately predict blue and brown eye colour with a precision of $>94 \%$, according to a previous study [9], using six of the most informative eye colour markers: rs12913832 (HERC2), rs1800407 (OCA2), rs12896399 (SLC24A4), rs16891982 (SLC45A2 (MATP)), rs1393350 (TYR) and rs12203592 (IRF4) in a single genotyping assay and a prediction model based on thousands of individuals for which IrisPlex genotype and eye colour phenotype data are available $[7,8,10]$. The $94 \%$ accuracy is based on using a threshold of $p>0.7$, however it is possible to use IrisPlex prediction with a lower $p>0.5$ threshold. The assessment of precision accuracy is based on a broad European dataset of $>3800$ individuals using IrisPlex can be found in Table 3 of that publication [9]. The IrisPlex assay represents the first FDP system that successfully underwent developmental validation using the Scientific Working Group on DNA Analysis Methods (SWGDAM) guidelines for use in forensic casework [10]. The IrisPlex prediction model, first established on thousands of Dutch Europeans, has been evaluated in several populations within and outside Europe and was shown to perform reliably, independent of the bio-geographic origin of the individual tested [9,11].

In an international effort to test the reliability and consistency of the IrisPlex system for eye colour prediction through an inter laboratory exercise, the European DNA Profiling (EDNAP) Group, a working group of the International Society for Forensic Genetics (ISFG), carried out a collaborative study led by the Department of Forensic Molecular Biology of the Erasmus University Medical Center Rotterdam (Netherlands), who initially developed and validated the IrisPlex system [8-10] and for this reason were chosen to conduct this further assessment on the IrisPlex tool alone. Of the 21 participating laboratories, 18 were from Europe, 
2 were from Australia and 1 was from the U.S.A. The prime aim of this exercise was to implement the method and assess the performance of the system across different forensic laboratories with varying levels of experience, from complete novices with no SNP typing experience to participants with SNaPshot experience and those with specific IrisPlex experience.

Notably, some authors previously raised issues about marker content and model outcomes of the IrisPlex system [12-15]. The present collaborate EDNAP exercise, however, represents a rather technical exercise to test the performance of the IrisPlex system across laboratories with varying levels of pre-existing experience. Therefore, issues about marker and model choice for predicting eye colour from DNA may be addressed in more dedicated future studies. Here, we present the results of this collaborative EDNAP exercise, placing emphasis on the reliability and consistency in using the IrisPlex system for blue and brown eye colour prediction from DNA.

\section{Materials and methods}

\subsection{Samples and materials provided to the participating laboratories}

The organising laboratory (Department of Forensic Molecular Biology, Erasmus MC University Medical Center Rotterdam, Netherlands) divided the entire exercise into three different tasks. All participants were provided with a detailed written laboratory protocol [8] as well as the eye colour prediction model that is an interactive excel spread-sheet as published earlier [10]. Furthermore, for assay interpretation guidelines, participants were given a protocol stating a 50 relative fluorescent units ( $\mathrm{rfu}$ ) peak height threshold should be used for allele calls using the IrisPlex specific GeneMapper software (Applied Biosystems) Bin and Panel set provided. For a broader understanding of average peak heights and balance ratios, participants were asked to refer to the previous developmental validation publication of the IrisPlex system [10]. In addition to the samples and the primers provided for each task, all reagents, which include: $1 \times$ PCR buffer, $2.7 \mathrm{mM} \mathrm{MgCl}_{2}, 200 \mathrm{mM}$ of each dNTP, $0.5 \mathrm{U}$ AmpliTaq Gold Polymerase, SNaPshot ${ }^{\mathrm{TM}}$ Multiplex chemistry for the single base extension (SBE) reactions (Applied Biosystems, Foster City, CA), Exonuclease Shrimp Alkaline Phosphatase (ExoSAP-IT), and Shrimp Alkaline Phosphatase (SAP) (USB Corporation, Cleveland, $\mathrm{OH}$ ), required for running the IrisPlex system were shipped on dry ice to each of the 21 participating laboratories. The laboratories were asked to use their own internal sizing standard (LIZ 120) and formamide for the capillary electrophoresis run.
Due to an ExoSAP-IT ${ }^{\mathrm{TM}}$ degradation issue noted during the early phase of the exercise, which subsequently was acknowledged by the producing company as a bad batch of enzyme, aliquots of a newly delivered and tested ExoSAP-IT ${ }^{\mathrm{TM}}$ were shipped again to the requesting laboratories, while the others opted to use their inhouse standard cleaning protocols. As this was a clean-up procedure, it did not impede on the testing of the IrisPlex assay overall. Purified products were run by the laboratories using their in-house Genetic Analysers (for type, see Table 1) and analysed with the previously published eye colour prediction model [10] provided by the organising laboratory for predicting human eye colour from IrisPlex genotypes.

As a disclaimer for the choice of samples used in this assessment, please note that it is well established and documented $[7,9,16,17]$, that the IrisPlex system through its use of six eye colour associated SNPs performs very well in predicting blue and brown eye colour with Area Under the receiving operator Curve (AUC) values $>0.9$; however its use for predicting intermediate eye colour (current AUC of $\sim 0.7$ ) is not at an optimum level yet. This is due to the current lack of knowledge on DNA predictors for these nonblue, non-brown eye colours i.e. green eye colour, individuals with heterochromia etc. which is not only a limitation of the IrisPlex but of all currently available DNA test systems for eye colour [4,6]. DNA variants with similarly high prediction effects on non-blue/brown eye colours as the IrisPlex SNPs have on blue and brown eye colour have yet to be identified. Therefore, the IrisPlex system was previously promoted for the prediction of blue and brown eye colours and thus the organising laboratory opted to test variations in blue and brown eye colour alone for Tasks 1 and 2 to evaluate the current IrisPlex system assay and prediction performance on these categories. Task 3 however incorporates all three categories as this task was based on samples provided by the participating laboratories who were not asked to focus on blue and brown eye colour alone when selecting their Task 3 volunteers.

Tasks 1 and 2 contain samples from individuals of European (80\% per task) and non-European bio-geographic origin (20\% per task) including one admixed individual in Task 1 . The individuals used in Task 3, including information about their bio-geographic background, were at the discretion of the participating laboratories and were unknown to the organising lab.

\subsection{Task 1 - IrisPlex eye colour prediction from biological samples with eye colour knowledge}

The organising laboratory provided all participating laboratories with five blood samples (labelled Ind1-Ind5) and five saliva

Table 1

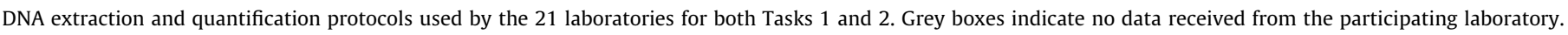

\begin{tabular}{|c|c|c|c|c|}
\hline Lab ID & Extraction Protocol & Quantification Protocol & Polymer & Genetic Analyzer \\
\hline 1 & & & $\mathrm{POP} 4$ & $3130 x \mathrm{xl}$ \\
\hline 2 & EZ1 DNA Investigator Kit on an EZ1 Advanced XL (Qiagen) & Quantifiler(ß) Duo DNA Quantification Kit (Life Technologies) & POP 6 & 3100 \\
\hline 3 & & & POP 4 & 3130 \\
\hline 4 & Task 1: EZl advanced (Qiagen); Task 3:DNA Blood Mini Kit (Qiagen) & ABI prism® 7900 (Life Technologies-LT) using Quantifiler $\mathbb{B}$ Human DNA Quantification Kit (LT). & POP 4 & $3130 x \mathrm{x}$ \\
\hline 5 & Chelex + Qiaquick & Task 1: Qubit and Task 2: RT-PCR Quantifiler(B) & POP 4 & $3130 \mathrm{xl}$ \\
\hline 6 & QIAampß DNA Mini (Qiagen, Hilden, Germany) & qPCR using the 7900HT Fast Real-time PCR System (Applied Biosystems, Darmstadt, Germany) and Alu Primers & POP 4 & $3500 x \mathrm{x}$ \\
\hline 7 & Chelex & Quantifilerß Duo & POP 7 & $3130 x \mathrm{x}$ \\
\hline 8 & $5 \%$ solution of BT Chelex 100 resin (Bio-Rad) & Quantifiler(1) Human DNA Quantification Kit (Life Technologies) on AB 7900 RT-PRC & POP 4 & 3130 \\
\hline 9 & QIAmp DNA blood Mini kit from Qiagen & Quantifiler( Human kit on Applied Biosystems 7500 Real-Time PCR System & POP 7 & $3500 x \mathrm{x}$ \\
\hline 10 & QIAmp DNA blood Mini kit from Qiagen & Nanodrop & POP 7 & $3500 \mathrm{xl}$ \\
\hline 11 & & Quantifilerß Human & POP 4 & $3130 \mathrm{xl}$ \\
\hline 12 & QIAamp mini columns (Qiagen) & real-time quantitative PCR assay using ALU repeats from Nicklas et el. & POP 7 & $3130 \mathrm{xl}$ \\
\hline 13 & EZ1 robot (Qiagen) & Quantifiler@ & POP 7 & $3130 x \mathrm{x}$ \\
\hline 14 & Qiagen EZ1 Advanced XL extraction robot with the EZI DNA Investigator Kit & Quantifiler@ Human DNA Quantification Kit (Life Technologies) & POP 4 & $3130 x \mathrm{x}$ \\
\hline 15 & QiAmp DNA Mini kit (Qiagen) & Nanodrop & POP 4 & $3130 \mathrm{xl}$ \\
\hline 16 & QiAmp DNA Mini kit (Qiagen) & & POP 7 & 3500 \\
\hline 17 & Qiagen EZ1 Investigator Handbook & Thermo Scientific NanoDrop 2000/2000c spectrophotometer & POP 4 & 3130 \\
\hline 18 & Applied Byosistems Prepfiler Forensic DNA Extraction kit & Task 1 and Task 3 a $1 \%$ agarose gel; Task 2 - a RT PCR & POP 4 & 3130 \\
\hline 19 & Chelex & Quantifiler@ DUO in an 7500 Real-Time PCR System & POP 7 & 3130 \\
\hline 20 & Phenol-Chloroform & Quantifiler@ Human DNA Quantification Kit and AB 7300. & POP 4 & 3130 \\
\hline 21 & Qiagen M48 robotic station and MagAttract DNA Mini M48 Kit & Quantifiler kitî and ABI 7500 & POP 7 & $3130 \mathrm{xl}$ \\
\hline
\end{tabular}


samples (labelled Ind6-Ind10) on FTA cards of 10 individuals with blue $(N=5)$ or brown $(N=5)$ eye colour. To produce these samples, fresh venous blood and saliva samples were collected from ten different individuals and $100 \mu \mathrm{L}$ of each of the samples were pipetted on to the FTA cards. A digital eye image from each of these 10 individuals who donated blood or saliva was also provided to the participants. All the laboratories were instructed to use their own in-house DNA extraction and quantification protocols. All participating laboratories were asked to generate the IrisPlex genotype profile from each of the 10 samples and using the provided IrisPlex prediction model, to conclude the eye colour prediction of the 10 donor individuals by noting the probability and precision accuracy per each sample and individual using the guidelines implemented in a previous publication [9] as provided to the participants. An example report was also provided to each laboratory to ease the fill-out for return. As the participants were provided with eye pictures, they already knew the eye colour of the study individuals of Task 1 before analyses. However, the conclusion of the eye colour phenotypes had to be based on the genotypes determined by each individual laboratory considering the probability and precision accuracy guidelines provided by the organising laboratory. The term 'precision accuracy' relates to the previous publication [9], which undertook a study on the final prediction called by the IrisPlex model in terms of probability values on over 3800 European individuals. It assesses the highest probability value (which is defined as the eye colour of the individual) and how correct the eye colour prediction was at thresholds that increase in increments of $0.05 \mathrm{p}$; from no threshold to $p>0.95$ [9].

\subsection{Task 2 - IrisPlex eye colour prediction from DNA of simulated casework samples without eye colour knowledge}

The laboratories were provided with five DNA samples extracted from simulated casework samples (labelled CW1CW5) from 5 individuals with blue $(N=2)$ or brown $(N=3)$ eye colour. DNA samples were extracted by the organising laboratory with the QIAamp DNA mini kit (Qiagen, Hagen, Germany). The following biological materials were used for DNA extractions: 2 buccal swabs (CW1 and CW2) - both samples subjected to UV radiation using the Bio-Link (Vilber Lourmat) for $1 \mathrm{~min}$ at a strength of $50 \mathrm{~J} / \mathrm{cm}^{2}$ before DNA extraction; saliva on glass slide (CW3) stored at room temperature for 1 week before DNA extraction, whole blood on glass slide (CW4) stored at room temperature for 1 week before DNA extractions, and semen DNA extracted from a frozen donated sample (CW5). DNA concentrations were measured using the nanodrop system and $3 \mu \mathrm{L}$ of the following solutions were provided: CW1: $0.5 \mathrm{ng} / \mu \mathrm{L}, \mathrm{CW} 2: 0.1 \mathrm{ng} /$ $\mu \mathrm{L}$, CW3: $0.25 \mathrm{ng} / \mu \mathrm{L}$, CW4: $2 \mathrm{ng} / \mu \mathrm{L}$, and CW5: $50 \mathrm{ng} / \mu \mathrm{L}$ (see Table 2 for overview). The samples CW1-CW4 were freshly extracted, quantified and run using the IrisPlex system prior to DNA degradation, thereby serving as a control to their degraded counterparts. In contrast to Task 1 , in Task 2 the laboratories were not provided with any eye colour phenotype information of the sample donors. Participants were also not provided with any other sample information such as DNA concentration or treatment prior

\section{Table 2}

Details of the simulated casework samples provided to the participating laboratories for the Task 2 .

\begin{tabular}{llll}
\hline Sample \# & Sample type & Treatment & Concentration $(\mathrm{ng} / \mu \mathrm{L})$ \\
\hline CW1 & Buccal Swab & UV for 1 min & 0.5 \\
CW2 & Buccal Swab & UV for 1 min & 0.1 \\
CW3 & Saliva on Slide & RT for 1 week & 0.25 \\
CW4 & Blood on slide & RT for 1 week & 2 \\
CW5 & Semen & - & 50 \\
\hline
\end{tabular}

to DNA extractions of the DNA extracts they received for Task 2. They were asked to generate the IrisPlex genotype profile for each individual and report back the obtained eye colour probabilities and accuracies using the model and materials provided, and to finally conclude the most likely eye colour category per individual. An example report was also provided.

\subsection{Task 3 - participant-driven IrisPlex eye colour prediction}

This part of the study was optional. Each participating laboratory was instructed to collect and genotype samples from five different individuals of any eye colour. Selection of volunteers and biological materials was at the discretion of the participants. An important caveat in this task is that IrisPlex cannot guarantee a high prediction accuracy of the non-blue and non-brown eye colours; however, in contrast to Tasks 1 and 2 no eye colour phenotype restrictions were imposed on the participants in their choice of volunteers for Task 3 . The laboratories were asked to report the DNA concentration, IrisPlex genotypes, eye colour probability outcomes and accuracy percentages, and a digital highresolution eye image of the genotyped individuals. The organising laboratory instructed that the iris photo should be taken in natural light conditions (no fluorescent bulb light) with and without flash lens using a digital camera focusing on eyes only (no full portrait).

\section{Results and discussion}

\subsection{Sample extractions and quantifications by each participating laboratory}

As the DNA extraction and quantification method can influence genotyping outcomes due to the quality and quantity of DNA extracted and consequently input into a downstream reaction, it can thus influence phenotype inference from the genotypes produced in FDP systems. Therefore we included extraction and quantification monitoring in this exercise. As part of Task 1, the host laboratory provided the participating laboratories with biological samples (blood and saliva samples on FTA cards) from which the participants extracted and quantified DNA using their methods of choice. To note, the affiliated laboratory number in the author list does not represent the laboratory number described throughout the paper. Protocols used for DNA extraction and quantification were different and are listed in Table 1. As evident, the different extraction and quantification methods used by the participating laboratories provided varying results, as summarised in a box plot diagram (Fig. 1), even though the same volume of biological sample was provided to each of the participants on FTA cards. Labs \#2, 4, 13, 14 and 17 used the Qiagen EZ1 investigator kit for extraction and reported on average higher quantification values as compared to Labs \#6, 9, 10, 12 and 15 that used the Qiagen QIAamp DNA mini kit extraction protocol. Lab \#20 applied a Phenol-Chloroform extraction approach, which yielded on average higher quantification values compared to all the other methods used. Lab \#18 used the Prepfiler Forensic DNA extraction kit and obtained on average higher final DNA amounts than all other methods, except Phenol-Chloroform. Labs \#5, 7, 8, and 19, which used the Chelex extraction protocol, reported comparatively lower quantification values than all other methods used in this exercise. Worthy to note, this figure assumes that all laboratory input DNA volumes were similar (i.e. the recommended $1 \mu \mathrm{L}$ ). The precise extraction volumes used by all labs were not available to the organising laboratory. This figure merely represents the differing extraction methods yielding varying final DNA concentrations, however, it is expected that the participants followed all recommendations provided by the organising lab which specifically states a $1 \mu \mathrm{L}$ volume with at least a concentration of $32 \mathrm{pg}$ 


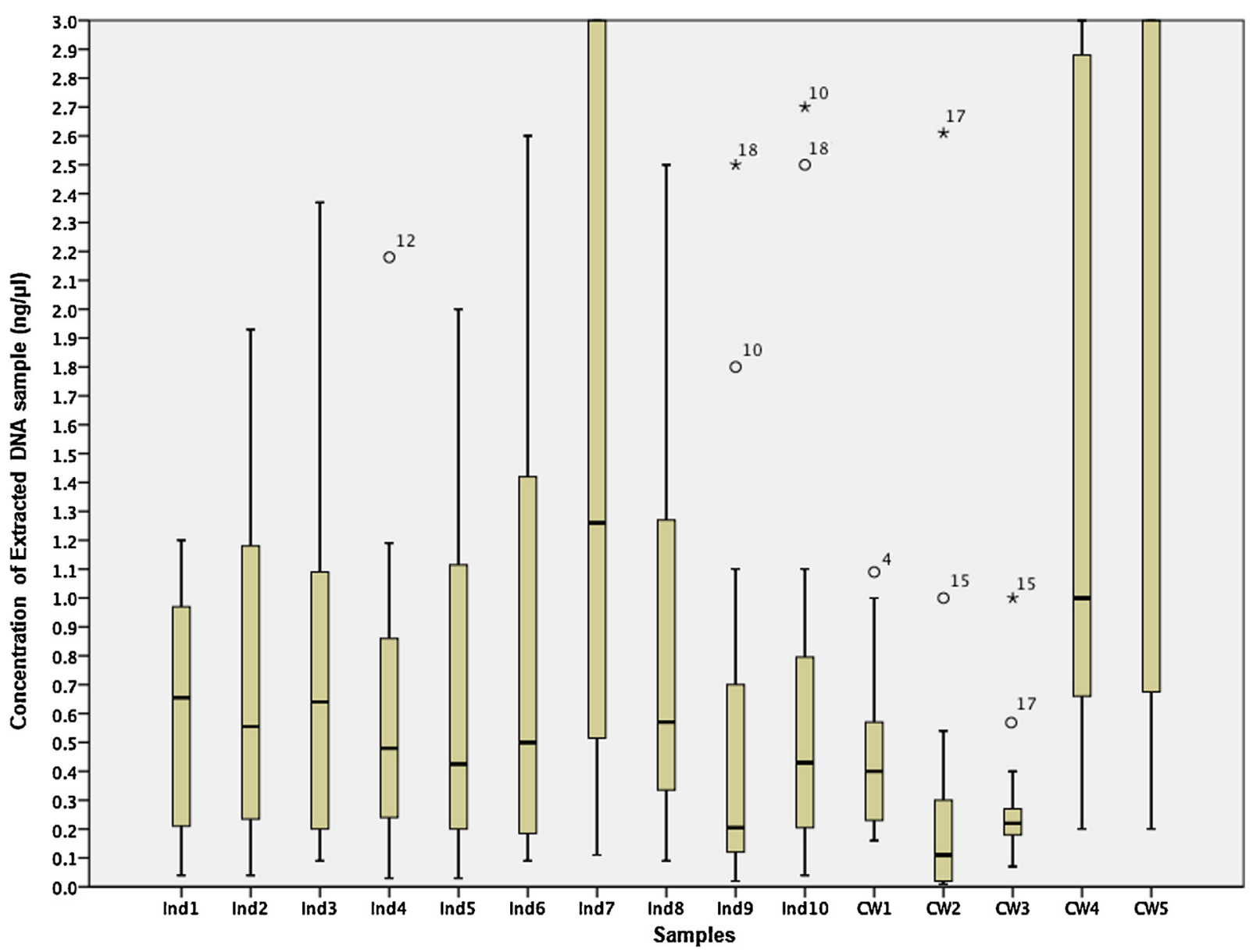

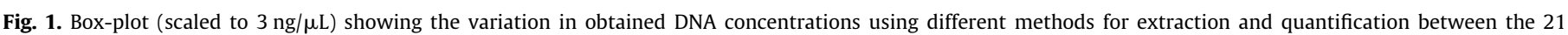

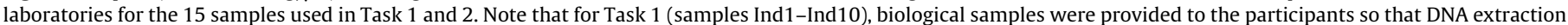

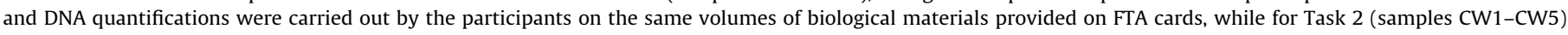

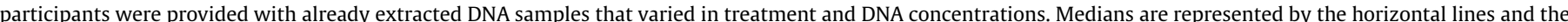

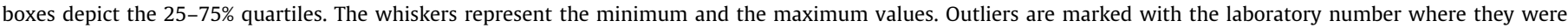
reported.

DNA input for IrisPlex profiling. The DNA samples provided for Task 2 were previously extracted by the host laboratory using the QIAamp DNA mini kit (Qiagen). The participating laboratories were requested to measure DNA concentrations using their method of choice and to report back the values. Because different quantification methods were used, the obtained concentration estimates differed (Fig. 1 and Supplementary Table 1), similar to Task 1, even though equal aliquots of the very same DNA solutions per each sample were provided to each of the participants. As evident, sample CW2 was recorded as the most variable $(0.01-2.61 \mathrm{ng} / \mu \mathrm{L})$, which contradicts recorded measurements by the organising laboratory of $100 \mathrm{pg}$ (Fig. 1).

Supplementary material related to this article can be found, in the online version, at doi:10.1016/j.fsigen.2014.04.006.

Overall, the recorded DNA quantification data indicate that all samples shipped, both the biological samples on FTA cards of Task 1 and the extracted DNA samples of Task 2, and remained rather stable during transportation and short-term storage at the participating laboratories. For the impact of the varying amounts of DNA obtained by the participants in Task 1 and the varying DNA concentration measures obtained in Task 2 on genotype and phenotype accuracy, see the specific chapters on Tasks 1 and 2 below. From the DNA quantification data reported by the participating laboratories for the samples used in Task 3 (Supplementary Table 1) it is evident that all the samples genotyped for this portion of the exercise were of reasonable quantity. When conducting genotyping analyses and calling the peaks, the $50 \mathrm{rfu}$ fluorescence threshold was set for calling alleles for a locus in all tasks and samples.

\subsection{Task 1 - IrisPlex eye colour prediction from biological samples with eye colour knowledge}

All participating laboratories reported the predicted eye colour and their probabilities in the format as requested by the organising laboratory. Fig. 2 depicts the accurate genotype and eye colour phenotype calls for all the ten samples as obtained by the 21 participating laboratories. Supplementary Table 2 lists the genotypes of the ten individuals with their respective eye colour probability and accuracy. Fig. 3 shows the eye colour images of the 10 individuals used in this task.

Supplementary material related to this article can be found, in the online version, at doi:10.1016/j.fsigen.2014.04.006.

Twenty of the 21 laboratories (95\%) predicted the eye colour of all 10 individuals included in Task 1 correctly from IrisPlex (Fig. 2; green bars). Overall, 208 (99\%) of the 210 samples analysed in this task by all the 21 laboratories were reported with the correct eye colour phenotype prediction. An overview of the samples with incorrect genotypes that were discordant with the organising laboratory is provided in Table 3. Only one laboratory (Lab \#3) faced difficulties in concluding the correct eye colour phenotype for two samples (Individual 1 and 10). The phenotype for both 


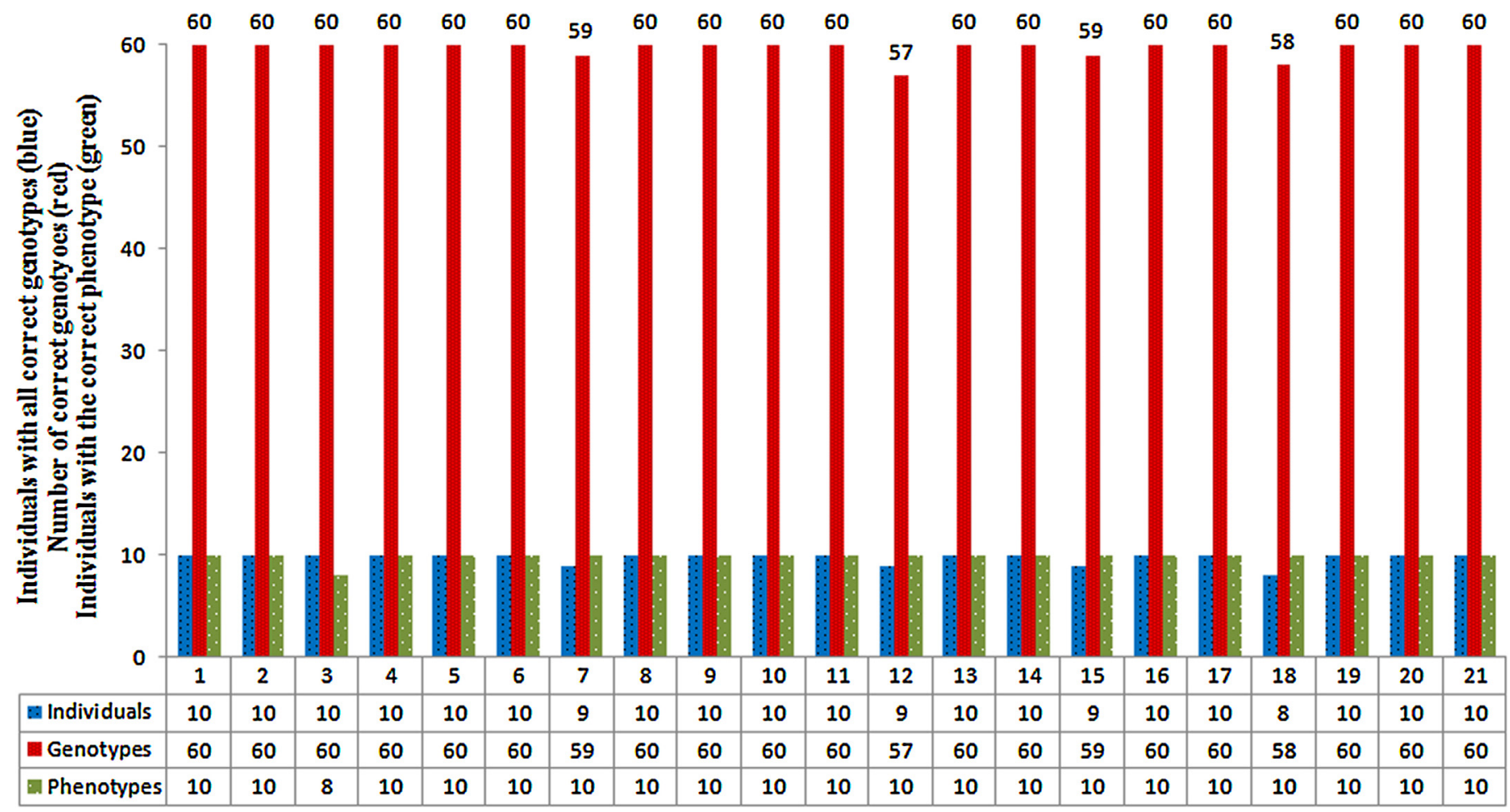

\section{Laboratory}

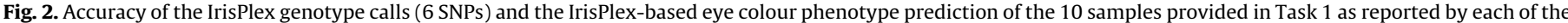

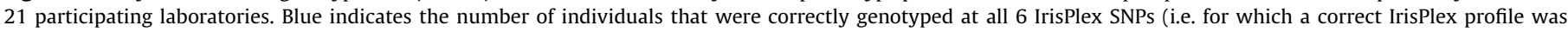

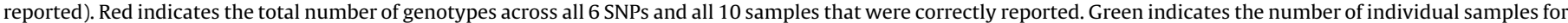

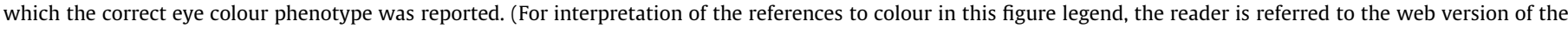
article.)

individuals was reported as inconclusive, although the correct IrisPlex genotypes were obtained and reported. These two individuals had eye colour probabilities for blue, intermediate and brown of $0.306,0.142,0.552$ and $0.299,0.253,0.448$ respectively (Fig. 3(a) and (k)), and did not cause a problem for the other 20 laboratories to conclude the correct brown eye colour for both samples.

Overall, 1253 (99.4\%) of the 1260 genotypes generated for the 6 IrisPlex SNPs in the 210 samples analysed by all the 21 laboratories were reported correctly. The $7(0.6 \%)$ incorrect genotypes were reported by 4 laboratories, while 17 participants reported the correct 6-SNP IrisPlex profiles for all 10 samples. Importantly, none of these incorrect genotypes led to erroneous eye colour phenotype predictions being reported. Lab \#7 reported an incorrect homozygous genotype $\mathrm{T}$ instead of the true heterozygous $\mathrm{CT}$ for rs 12203592 for Individual 8 due to a dropout of the $C$ allele, since the respective peak was below the $50 \mathrm{rfu}$ threshold. Lab \#12 reported discordant heterozygous instead of correct homozygous genotypes for Individual 9 across three markers (rs12913832, rs16891982 and rs1393350). Drop-in of the alleles C, G and T was observed for each of the markers rs12913832, rs16891982 and rs1393350 respectively. However, the DNA concentration for this particular sample was reported by Lab \#12 to be very low (0.02 ng/ $\mu \mathrm{L}$ ), much lower than the concentration obtained by the other

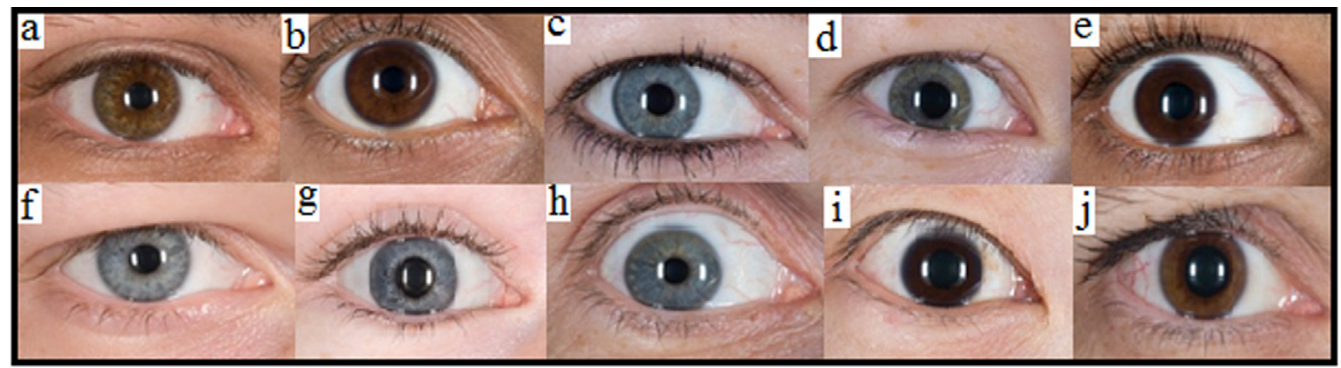

Task 2

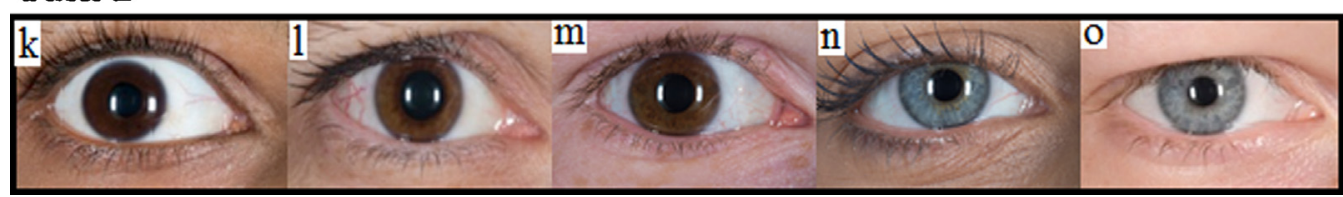

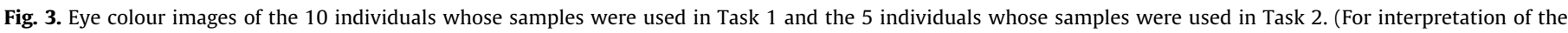
references to colour in this figure legend, the reader is referred to the web version of the article.) 
Table 3

Tasks 1 and 2 genotyping results that were discordant between the host and the participating laboratories.

\begin{tabular}{llcll}
\hline & Sample & Lab\# & Locus & Comments \\
\hline Task 1 & Individual 8 & 7 & rs12203592 & Drop-out of C \\
& Individual 9 & 12 & rs12913832 & Drop-in of C \\
& Individual 9 & 12 & rs16891982 & Drop-in of G \\
& Individual 9 & 12 & rs1393350 & Drop-in of T \\
& Individual 2 & 15 & rs16891982 & Drop-out of C \\
& Individual 3 & 18 & rs12203592 & Drop-in of T \\
& Individual 8 & 18 & rs12203592 & Drop-out of T \\
Task 2 & CW2 & 6 & rs12913832 & Drop-out of T \\
& CW2 & 7 & rs12913832 & Drop-out of C \\
& CW2 & 15 & rs1393350 & Drop-in of T \\
& CW2, CW3 & 17 & rs12913832 & Drop-out of T, \\
& & & & C respectively \\
& CW2 & 17 & rs1800407 & Drop-out of A \\
& CW3 & 18 & rs1393350 & Drop-out of T \\
& CW2 & 21 & rs12896399 & Drop-out of T \\
\hline
\end{tabular}

laboratories for this sample (Supplementary Table 1). In accordance with the provided protocol, $1 \mu \mathrm{L}$ of DNA solution of this sample was used for the IrisPlex PCR. Therefore, for this sample the amount of DNA input was below the previously established sensitivity threshold of the IrisPlex assay [10], which explains the high failure rate for genotypes of this sample. Lab \#15 reported incorrect homozygous genotype of the $G$ allele (instead of the correct heterozygous GC genotype) for individual 2 at rs16891982 due to a dropout of the $C$ allele. Lab \#18 experienced at marker rs12203592 a drop-in of allele $\mathrm{T}$ for individual 3 and dropout of allele $\mathrm{T}$ in individual 8. One explanation could be primer degradation due to incorrect storage of the primer or an incorrect volume addition of this primer to the assay, but unlikely due to a sample issue as the DNA concentrations reported for the individuals 2,3 and 8 by the Labs \#15 and 18 were more than $1.1 \mathrm{ng} / \mu \mathrm{L}$ (provided that the correct input of $1 \mu \mathrm{L}$ was used). For cases such as these, an erroneous result can be avoided by re-running several analyses of the sample, and is usually recommended when using all genotyping platforms, this includes the IrisPlex system.

\subsection{Task 2 - IrisPlex eye colour prediction from DNA of simulated} casework samples without eye colour knowledge

In Task 2, the provided five DNA extraction aliquots from simulated casework samples (Table 2) were evaluated by each participating laboratory. Notably, the samples used for DNA extractions not only came from different biological sources (saliva, blood, and semen) but also experienced different environmental conditions (UV radiation, storage at room temperature) and were provided to the participants in varying DNA concentrations (0.1$50 \mathrm{ng} / \mu \mathrm{L}$ ), all unknown to the participants. Therefore, and due to the fact that no eye colour phenotypes were provided of the sampled individuals, this task was more challenging than Task 1 . Fig. 4 depicts the accurate genotype and eye colour phenotype calls for all the 5 samples as reported by the 21 participating laboratories. Supplementary Table 2 shows the reported genotypes of the five samples with their respective eye colour prediction probabilities and precision accuracy. Fig. 3 shows the eye colour images of the 5 individuals used in this task. An overview of the samples with incorrect genotypes that were discordant with the organising and the other participating laboratories is provided in Table 3.

Eighteen (86\%) of the 21 laboratories predicted the eye colour of all 5 individuals correctly from IrisPlex (Fig. 4; green bars). Overall, 101 (96.2\%) of the 105 samples analysed by all the 21 laboratories together were reported with the correct eye colour phenotype. The 4 samples (3.8\%) for which the eye colour phenotypes were incorrect had been reported by 3 laboratories. Lab \#3 predicted the eye colour of 2 of the 5 individuals (CW2 and CW3) as inconclusive, although the genotypes for these samples were reported correctly. Both samples clearly had to be designated as brown from the obtained probabilities ( $p=0.448$ and $p=0.552$, respectively), and the phenotypes indeed were brown (Fig. $3(1)$ and ( $\mathrm{m}$ ) respectively), as was correctly interpreted by 18 other laboratories. The other 2 incorrectly phenotyped samples were reported by Labs \#6 and 17 due to the drop-out of the T allele at rs12913832 in sample CW2. The 2 laboratories reported an incorrect homozygous $C$ allele instead of a heterozygous CT allele, thereby, reporting an incorrect blue eye colour instead of the correct brown eye colour phenotype (Fig. 3(1)).

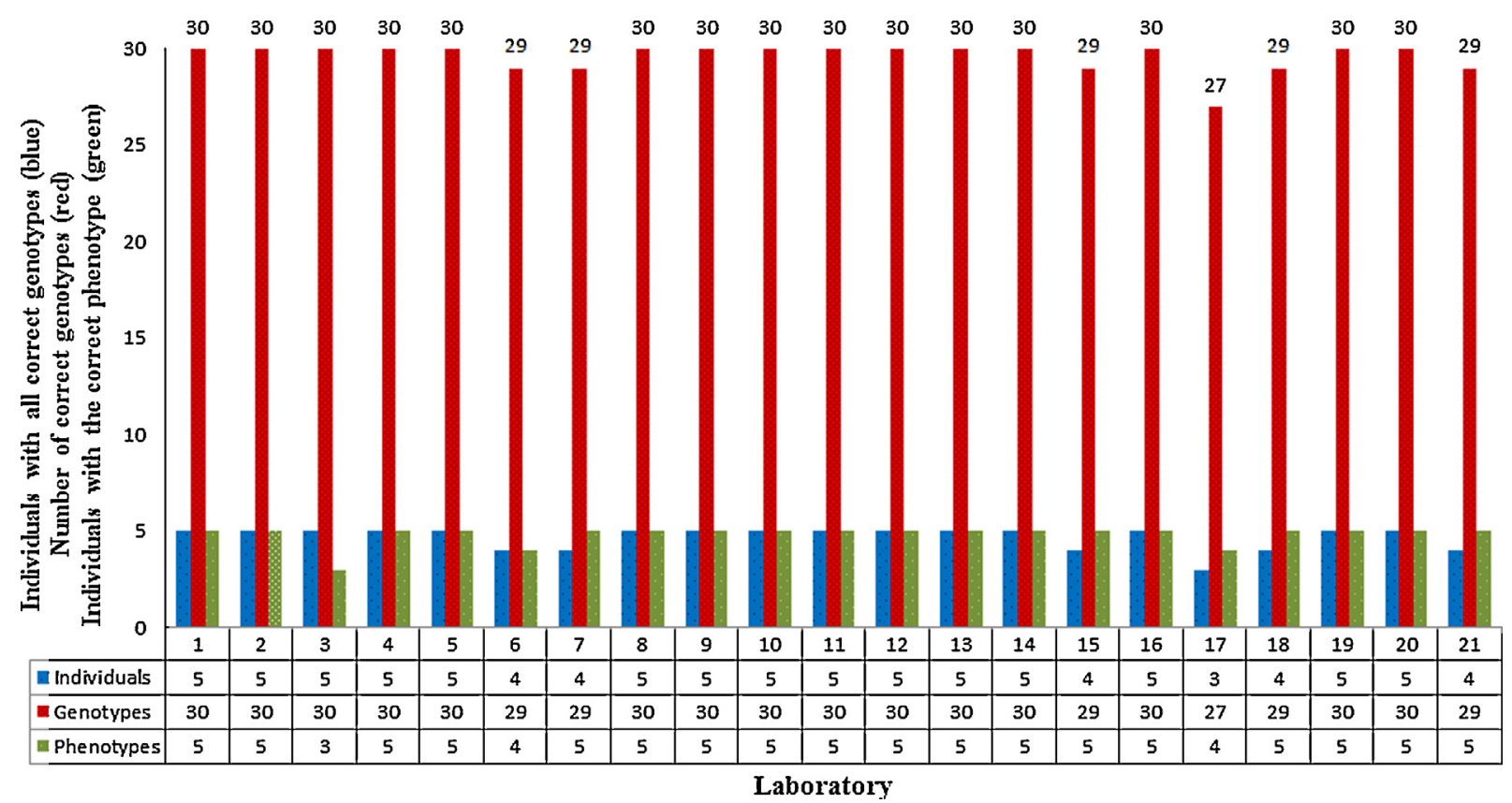

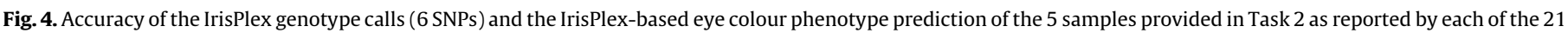

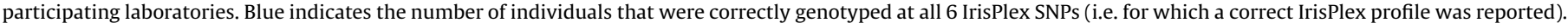

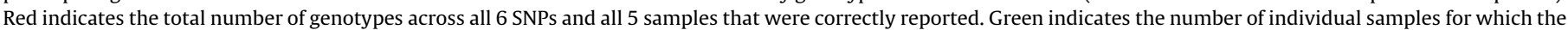

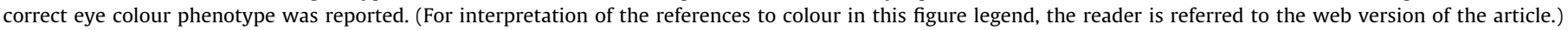


Overall, 622 (98.7\%) of the 630 genotypes generated for the 6 IrisPlex SNPs in the 105 samples analysed by all laboratories together were correctly reported in Task 2 . The 8 (1.3\%) incorrect genotypes were produced in 2 samples (CW2 and CW3) by 6 laboratories, while 15 of the 21 laboratories (71.4\%) reported the correct 6-SNP IrisPlex profile for all 5 samples. In contrast to the 2 (25\%) genotype errors in sample CW2 by Lab \#6 and 17 that caused phenotype errors as mentioned in the previous paragraph, the remaining 6 incorrect genotypes (75\%) did not have any impact on the eye colour phenotype accuracy. At rs12913832, Lab \#7 reported an incorrect genotype due to a drop-out of the $C$ allele for sample CW2. A drop-in of the T allele for CW2 and drop-out of the T allele for CW3 at rs1393350 resulted in incorrect genotyping by Labs \#15 and 18 respectively. Furthermore, incorrect genotypes were reported by Lab \#21 at rs12896399 for sample CW2 due to a drop-out of the T allele. Lab \#17 experienced problems in the first typing of samples CW1, 2 and 3 and subsequently retyped these samples in different DNA dilutions. At rs12913832, drop-out of the T allele for CW2 (as mentioned above); drop-out of the $C$ allele for CW3, and drop-out of the A allele at rs1800407 for CW2 were reported which resulted in erroneous results for this laboratory. A dilution step performed by the participating laboratory, due to a misleading quantification result, of the already low quantity degraded samples provides a likely explanation for the drop-out of the alleles in this set of samples.

Several laboratories ( $n=3$; Labs \#3, 6 and 17) experienced difficulties with correct phenotyping of the simulated and treated casework samples in Task 2 for which no eye colour phenotypes were provided as opposed to the untreated biological samples provided together with eye colour phenotypes in Task 1 ( $n=1$; Lab \#3). Similarly, more laboratories ( $n=6$; Labs \#6, 7, 15, 17, 18 and 21) had difficulties in correct genotyping of Task 2 samples in relation to Task 1 samples ( $n=4$; Labs \#7,12, 15 and 18). Within Task 2, the most genotyping and phenotyping difficulties i.e. allelic drop-outs and drop-ins were reported for 2 particular samples (CW2 and CW3). Sample CW2 was reported with different incorrect genotypes by 5 of the laboratories (Labs \#6, 7, 15, 17 and 21) and sample CW3 was reported incorrectly by 2 laboratories (Labs \#17 and 18) (see Table 3 for overview). Sample CW2 must therefore be noted as being a difficult sample to genotype. From Fig. 1, it is evident that, of the laboratories that reported quantification data for Task 2 , sample CW2 was recorded as the most variable $(0.01-2.61 \mathrm{ng} / \mu \mathrm{L})$, which strongly deviates from the recorded measurements by the organising laboratory of $100 \mathrm{pg}$. Given its unusual quantification range, severe degradation and heterozygosity at 3 (rs12913832, rs1800407 and rs12896399) of the 6 SNPs, increased incidence of allelic drop-out may be expected in sample CW2 as compared to the homozygous sample CW1 (which also experienced UV degradation) that caused no problems for genotyping. This demonstrates, as expected and as also known for any other genotyping assay, that the combination of low quality and low quantity template DNA provides challenges for correct genotyping including for the IrisPlex assay. However, it should be emphasised that 244 of the 252 (96.8\%) genotypes of the most challenging samples CW2 and CW3 were generated correctly by 15 of the 21 (71.4\%) participating laboratories, which demonstrates the reliability of the IrisPlex assay for difficult DNA samples. This also represents the necessity of employing duplicate analysis when genotyping samples of low DNA quantity in final case work applications.

\subsection{Task 3 - participant-driven IrisPlex testing}

The optional Task 3 of the exercise, where participants were asked to recruit their own volunteers for IrisPlex genotyping and eye colour prediction, was performed by 20 of the 21 laboratories.
Lab \#7 could not perform this task due to reported ethical issues. Supplementary Table 3 summarises the data for this task. Based on the digital eye images provided by the participants (Fig. 5), the organising laboratory judged the correct phenotypes by two independent experienced observers. Lab \#12 performed this exercise and reported the genotype and phenotype, but provided no eye images to the host laboratory for inspection. As it was not possible to judge the accuracy of the results provided by this participant, they were excluded from the analyses. As can be seen in Fig. 6, 16 of the 19 laboratories (84.2\%) predicted the eye colour of all analysed individual samples correctly, while 3 laboratories faced difficulties in concluding the correct eye colour from the estimated probability combinations for some samples. Overall, 96 (96\%) of the 100 samples analysed by the 19 laboratories were reported with the correct eye colour prediction, as judged by the organising laboratory based on the digital eye images sent by the participants. The 4 samples reported with incorrect eye colour were from 3 different laboratories ( $1 x \# 2,2 x \# 3$, and 1x\#5). Lab \#2 reported blue eye colour $(p=0.678)$ for their sample 1 but the eye image showed brown colour and the estimated brown eye probability was only 0.191 (Fig. 5(a)). Lab \#3 obtained the following probabilities for their sample 1 : Blue -0.207 , Intermediate -0.161 and Brown -0.632 and reported an inconclusive result, while the probability for brown was by far higher than for the other two categories so that brown should have been concluded instead and indeed the respective eye image showed brown (Fig. 5(b)). Sample 4 of Lab \#3 appeared blue from the images (Fig. 5(c)) but a high brown eye probability ( $p=0.892)$ was obtained while the blue eye probability was low $(p=0.024)$. Lab \#5 obtained probabilities of Blue -0.375 , Intermediate -0.264 and Brown -0.361 for their sample 1 , and reported blue eye colour but the image indicates brown eye colour (Fig. 5(d)). In this case, however, the eye colour could have been reported inconclusive since the brown and the blue eye colour probabilities were very similar. It is therefore important to use and report the level of precision accuracy based on each probability threshold with the final prediction, i.e. $p<0.5$ highest probability value, $p>0.5$ highest probability value. This can be found in Table 2 of our previous publication [9]. It is noteworthy to emphasise that the IrisPlex genotypes in Task 3 were not verified independently in contrast to those in Tasks 1 and 2. Therefore we cannot know for sure if any of the 4 incorrect phenotype predictions in Task 3 may have been caused by incorrect genotypes, although the high genotyping accuracy rates achieved in Tasks 1-3 suggest this might be somewhat unlikely. Due to violation of anonymity, the geographic origin of these individuals cannot be determined.

Supplementary material related to this article can be found, in the online version, at doi:10.1016/j.fsigen.2014.04.006.

Further to note, participants for Task 3 were not asked to restrict their choice of volunteers to blue and brown eye colour only. This was different from Tasks 1 and 2 where only volunteers with blue and brown eye colour were used due to known limitations of the IrisPlex system to accurately predict non-blue and non-brown eye colours [8-10]. However only Lab \#1 reported 2 individuals as intermediate $(p=0.411$ and $p=0.405)$ and from the eye images (Fig. 5(e) and (f) respectively), we can confirm that the individuals were correctly predicted as intermediate as they contain substantial pupillary rings of a different colour (i.e. majority of iris blue colour with obvious brown pupillary ring). It is also worth noting that although no restrictions were imposed on the choice of samples for Task 3, all the laboratories (except Lab \#1) chose individuals with either blue or brown eyes and hence it is most likely that all the participants were guided by knowledge of (or clearly considered the) limitations of IrisPlex for accurately predicting non-blue and non-brown eye colour phenotypes. Furthermore, according to general knowledge, the frequency of 

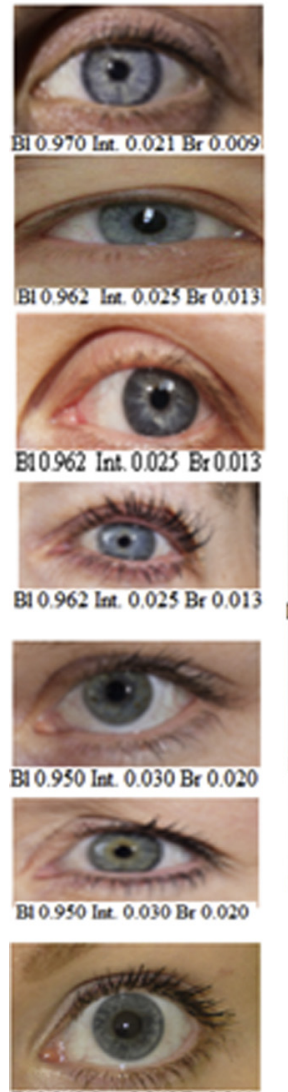

B10945 Int, 0.045 Br 0.011
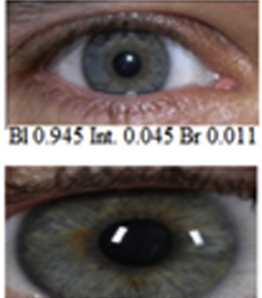

BIUYSI Int U.U4IBruU:L2

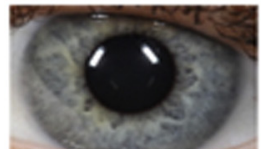

B10937 In: $0041 \mathrm{Br} 0022$

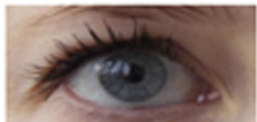

B1 0.937 Int $0.041 \mathrm{Br} 0.022$

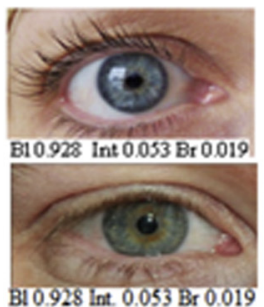

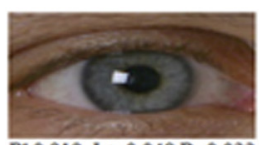

Bd 0.919 Itat. 0.048 Br 0.033

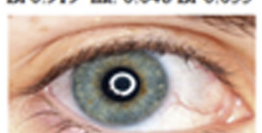

B10919 Int 0048 B. 0.033

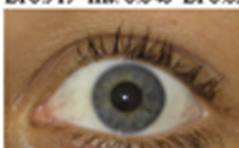

B10919 Imt 0048 Br 0033

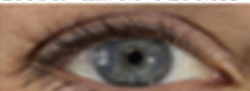

B1 0.919 Int. $0.048 \mathrm{Br} 0.033$

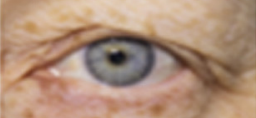

Bd0.919 Ine. 0.048 Br 0.033

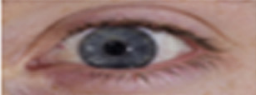

Br0.919 Int. $0.048 \mathrm{Br} 0.033$

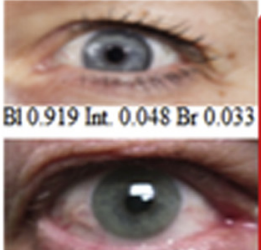

Bt 0.919 Int $0.048 \mathrm{Br} 0.03$

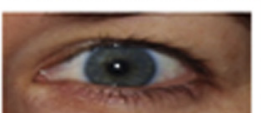

10.908 Int. $0.073 \mathrm{Br} 0.020$

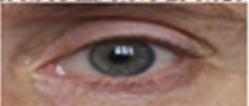

3 $0 . \$ 99$ Int $0.066 \mathrm{Br} 0.035$
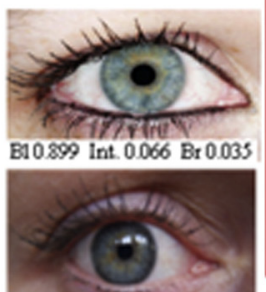

B10.899 Int. 0.066 Br 0.035

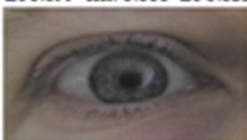

H10.859 Int 0.005 Dr 0.09

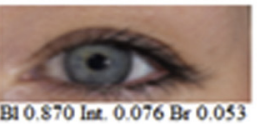

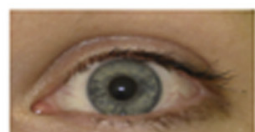
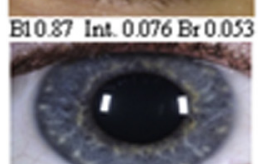

B10.87 Int. $0.076 \mathrm{Br} 0.053$
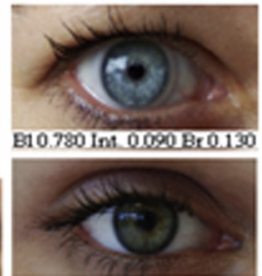

Bd 0.774 Int $0.081 \mathrm{Br} 0.145$
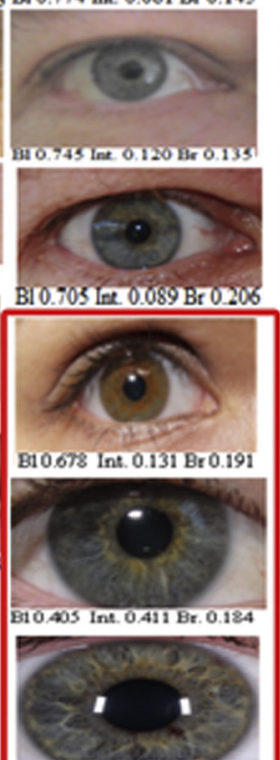

B10.375 Int. 0.264 Br 0361

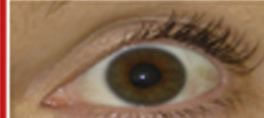

B10.375 Int. 0.264 Br 0.36
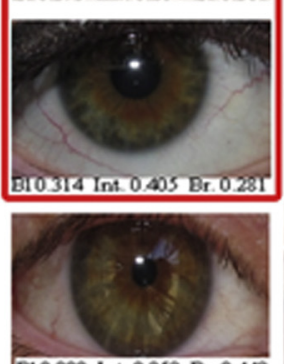

B10.299 Int. 0.253 Br. 0.443

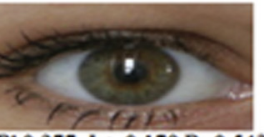

B1 0.277 Int. 0.179 Br 0.54

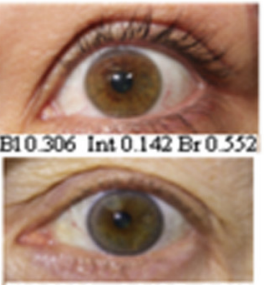

Bd 0.306 Int $0.142 \mathrm{Br} 0.552$

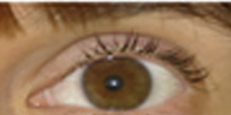

B10.185 Int. 0201 Bt 0.614

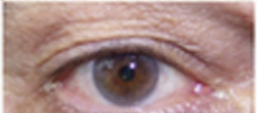

B10.207 Int $0.161 \mathrm{Br} 0.632$

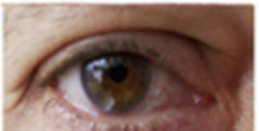

B10.207 Int. 0.161 Br 0.632

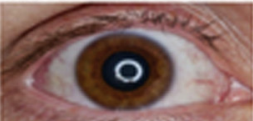

B10.207 Int $0.161 \mathrm{Br} 0.632$

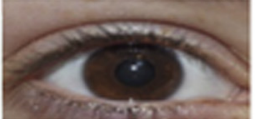

890.229 Ine $0.128 \mathrm{Br} 0.643$

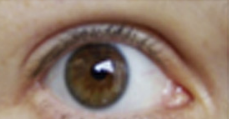

B10.134 Int $0.175 \mathrm{Br} 0.69$

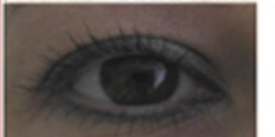

Bi 0.134 Ins. $0.175 \mathrm{Br} 0.69$
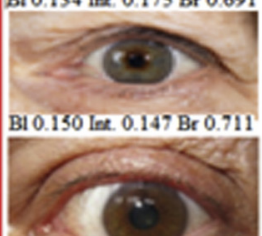

B10.15 Int 0.14 Br 0.71
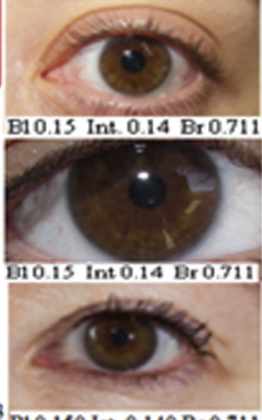

B1 0.130 Int 0.140 B4 0.71
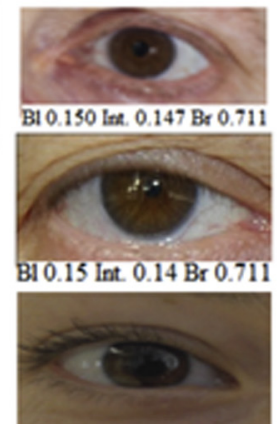

990.094 Int 0.148 Br 0.758

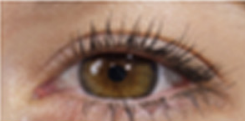

B4 0.094 Int 0.148 Br 0.758

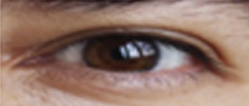

B1 0.040 Int. $0.151 \mathrm{Br} 0.81$
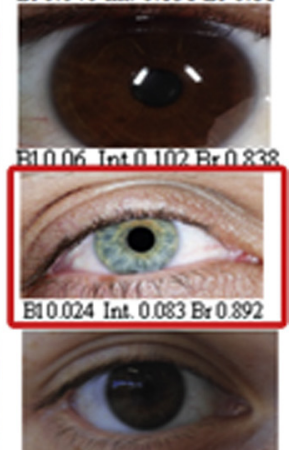

210.016 int. $0.043 \mathrm{Br} 0.941$

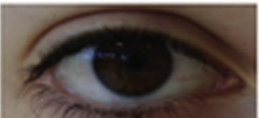

B10016 Int $0.043 \mathrm{Br} 0.941$

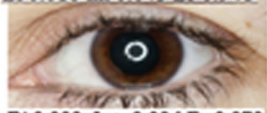

B10002 Int $0026 \mathrm{Br} 0.972$
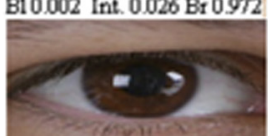

Bg 0.001 Int $0.025 \mathrm{Br} 0.974$

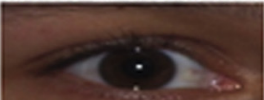

BI 0 Int. $0.013 \mathrm{Br} 0.987$

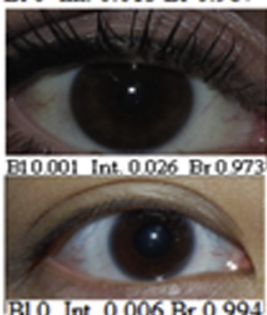

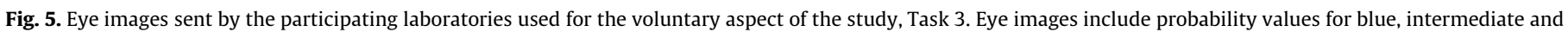

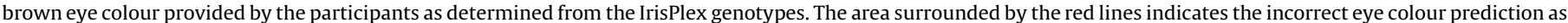

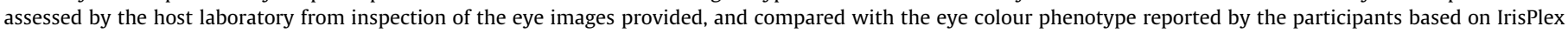
analysis. (For interpretation of the references to colour in this figure legend, the reader is referred to the web version of the article.) 


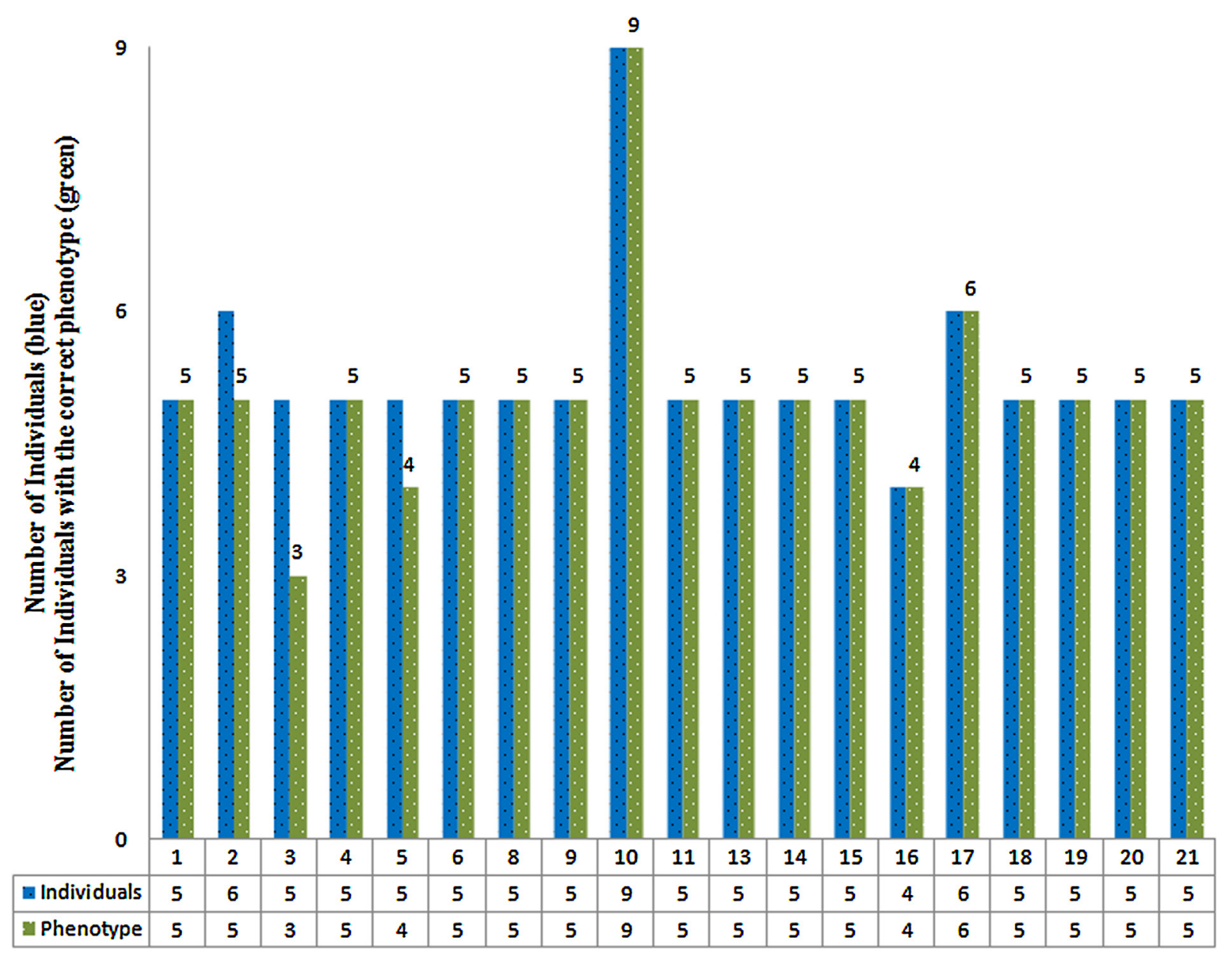

Laboratory

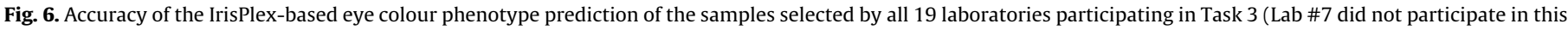

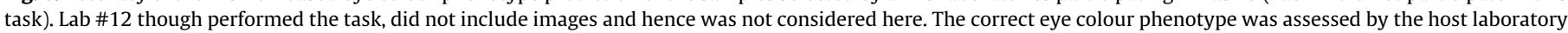

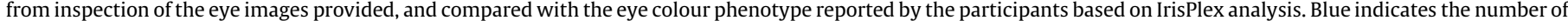

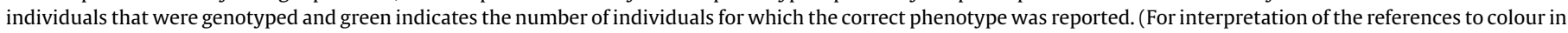
this figure legend, the reader is referred to the web version of the article.)

blue and/or brown eyes is comparatively higher than intermediate in the population, which could explain the rarity of volunteers with intermediate eye colour phenotype used in Task 3.

The overall performances of the participating laboratories in all 3 tasks, is shown in Supplementary Table 4.

Supplementary material related to this article can be found, in the online version, at doi:10.1016/j.fsigen.2014.04.006.

\section{Conclusions}

Overall, the high level of consistency achieved throughout this collaborative effort in all 3 tasks illustrates the reliability of the IrisPlex assay in producing highly accurate 6-SNP genotypes and of the IrisPlex prediction model in producing accurate blue and brown eye colour phenotypes from IrisPlex genotypes. As shown here and previously [8], the IrisPlex assay provides reproducible results despite differing levels of experience of the laboratory personnel involved and differing DNA extraction and quantification methods used. The results obtained in this collaborative exercise demonstrate the robustness and reproducibility of DNAbased eye colour prediction when using the IrisPlex system in different forensic laboratories world-wide. As emphasised before [8-10], future focus shall be placed on improving DNA-based prediction of non-blue and non-brown eye colours, for which the
IrisPlex system is less suitable than for blue and brown eye colour prediction from DNA.

\section{Acknowledgements}

We would like to thank Gabriela Huber, Nelli Roininen and Mintu Hedman for outstanding technical assistance and Vlastimil Stenzl for support. This work was funded in part by the Netherlands Forensic Institute (NFI) and a grant from the Netherlands Genomic Initiative (NGI)/Netherlands Organization for Scientific Research (NWO) within the framework of the Forensic Genomics Consortium Netherlands (FGCN). Funding for Adrian Linacre and Renee Ottens was provided by the Attorney General's Office, South Australia, for Theresa Gross by the EU 7th Framework Programme (FP7/2007-2013) under Grant Agreement $\mathrm{N}^{\circ} 285487$ (EUROFORGEN-NoE), and for Antti Sajantila by the Finnish Foundations' Pool Professorship (Paulo Foundation).

\section{References}

[1] M. Kayser, P.M. Schneider, DNA-based prediction of human externally visible characteristics in forensics: motivations, scientific challenges, and ethical considerations, Forensic Sci. Int. Genet. 3 (June (3)) (2009) 154-161.

[2] M. Kayser, P. de Knijff, Improving human forensics through advances in genetics, genomics and molecular biology, Nat. Rev. Genet. 12 (March (3)) (2011) 179-192. 
[3] J. Han, P. Kraft, H. Nan, Q. Guo, C. Chen, A. Qureshi, S.E. Hankinson, F.B. Hu, D.L. Duffy, Z.Z. Zhao, N.G. Martin, G.W. Montgomery, N.K. Hayward, G. Thomas, R.N. Hoover, S. Chanock, D.J. Hunter, A genome-wide association study identifies nove alleles associated with hair color and skin pigmentation, PLoS Genet. 4 (May (5)) (2008) e1000074.

[4] Y. Ruiz, C. Phillips, A. Gomez-Tato, J. Alvarez-Dios, M. Casares de Cal, R. Cruz, O. Maroñas, J. Söchtig, M. Fondevila, M.J. Rodriguez-Cid, A. Carracedo, M.V. Lareu, Further development of forensic eye color predictive tests, Forensic Sci. Int. Genet. 7 (January (1)) (2013) 28-40.

[5] G.G. Fortes, C.F. Speller, M. Hofreiter, T.E. King, Phenotypes from ancient DNA: approaches, insights and prospects, Bioessays 35 (August (8)) (2013) 690-695.

[6] K.L. Hart, S.L. Kimura, V. Mushailov, Z.M. Budimlija, M. Prinz, E. Wurmbach, Improved eye- and skin-color prediction based on 8 SNPs, Croat. Med. J. 54 (June (3)) (2013) 248-256.

[7] F. Liu, K. van Duijn, J.R. Vingerling, A. Hofman, A.G. Uitterlinden, A.C. Janssens, M. Kayser, Eye color and the prediction of complex phenotypes from genotypes, Curr. Biol. 19 (March (5)) (2009) R192-R193.

[8] S. Walsh, F. Liu, K.N. Ballantyne, M. van Oven, O. Lao, M. Kayser, IrisPlex: sensitive DNA tool for accurate prediction of blue and brown eye colour in the absence of ancestry information, Forensic Sci. Int. Genet. 5 (June (3)) (2011) 170-180.

[9] S. Walsh, A. Wollstein, F. Liu, U. Chakravarthy, M. Rahu, J.H. Seland, G. Soubrane, L. Tomazzoli, F. Topouzis, J.R. Vingerling, J. Vioque, A.E. Fletcher, K.N. Ballantyne, M. Kayser, DNA-based eye colour prediction across Europe with the IrisPlex system, Forensic Sci. Int. Genet. 6 (May (3)) (2012) 330-340.
[10] S. Walsh, A. Lindenbergh, S.B. Zuniga, T. Sijen, P. de Knijff, M. Kayser, K.N. Ballantyne, Developmental validation of the IrisPlex system: determination of blue and brown iris colour for forensic intelligence, Forensic Sci. Int. Genet. 5 (November (5)) (2011) 464-471.

[11] J. Purps, M. Geppert, M. Nagy, L. Roewer, Evaluation of the IrisPlex eye color prediction tool in a German population sample, Forensic Sci. Int. Genet. 3 (December (1)) (2011) 202-203.

[12] C. Martinez-Cadenas, M. Peña-Chilet, M. Ibarrola-Villava, G. Ribas, Gender is a major factor explaining discrepancies in eye colour prediction based on HERC2 OCA2 genotype and the IrisPlex model, Forensic Sci. Int. Genet. 7 (July (4)) (2013) 453-460.

[13] P.R. Prestes, R.J. Mitchell, R. Daniel, K.N. Ballantyne, R.A.H. van Oorschot, Evaluation of the IrisPlex system in admixed individuals, Forensic Sci. Int. Genet. 3 (December (1)) (2011) e283-e284.

[14] G.M. Dembinski, C.J. Picard, Evaluation of the IrisPlex DNA-based eye color prediction assay in a United States population, Forensic Sci. Int. Genet. 9 (March) (2014) 111-117.

[15] L. Yun, Y. Gu, H. Rajeevan, K.K. Kidd, Application of six IrisPlex SNPs and comparison of two eye color prediction systems in diverse Eurasia populations, Int. J. Legal Med. (January) (2014), http://dx.doi.org/10.1007/s00414-013-0953-1.

[16] V. Kastelic, E. Pośpiech, J. Draus-Barini, W. Branicki, K. Drobnič, Prediction of eye color in the Slovenian population using the IrisPlex SNPs, Croat. Med. J. 54 (August (4)) (2013) 381-386.

[17] S. Walsh, F. Liu, A. Wollstein, L. Kovatsi, A. Ralf, A. Kosiniak-Kamysz, W. Branicki, M. Kayser, The HIrisPlex system for simultaneous prediction of hair and eye colour from DNA, Forensic Sci. Int. Genet. 7 (January (1)) (2013) 98-115. 